\title{
Dispositivos Móviles en Educación Superior: la experiencia con Kahoot!
}

\author{
Gerusa Giménez Leal y Rodolfo de Castro Vila
}

Recibido: 15 de Febrero de 2019

https://doi.org/10.37610/dyo.v0i70.565

Aceptado: 13 de Marzo de 2019

\begin{abstract}
Resumen
En los últimos años han proliferado las herramientas para gamificar el aprendizaje en el aula en forma de aplicaciones para dispositivos móviles. El presente trabajo analiza diferentes herramientas de gamificación, en base a dispositivos móviles, y propone una clasificación según sus objetivos educacionales o ámbito de utilidad. El trabajo también realiza una propuesta metodológica de gamificación a través del uso de la aplicación Kahoot! en los estudios de Ingeniería. Los resultados de esta experiencia muestran como la participación activa de los estudiantes se estimula y aumenta su motivación e interés en la materia.
\end{abstract}

\section{Palabras clave}

Gamificación, Ingeniería, Dispositivos móviles, Educación 3.0, Kahoot!

\section{Introducción}

La adopción del concepto Gamificación o Game-Based Learning (GBL) en la docencia universitaria es cada vez más frecuente (Prieto et al, 2014). Este concepto surge de relacionar las estrategias del juego y el uso de la tecnología como una metodología para aplicar en el contexto educativo (Deterding et al, 2011; Prensky, 2001).

Esta se demuestra muy útil como mecanismo para aumentar la motivación y la participación del alumnado en clase (Carnevale, 2005; Hamari et al, 2014). Muchas experiencias de gamificación se basan en el uso de videojuegos, juegos de mesa, concursos de preguntas o simulaciones de entornos reales. El hecho que los dispositivos móviles (smartphones o tablets) se hayan convertido en una extensión de nosotros mismos y en una herramienta fundamental para gestionar infinidad de tareas de nuestro día a día, ha llevado a introducirlos también en el ámbito de la enseñanza superior. El trabajo de López y Silva (2016) afirma que el 75\% de los estudiantes universitarios utilizan dispositivos móviles en actividades asociadas a sus estudios. Otros trabajos demuestran que su uso en el aula también puede contribuir en la mejora del proceso de enseñanza-aprendizaje (Papastergiou, 2009; Shell, 2013; Wang et al, 2016) proporcionando nuevas herramientas a los docentes y ayudando en la adquisición de conceptos entre los estudiantes, especialmente a los llamados "nativos digitales”, muy acostumbrados al uso de la tecnología (Bennett et al, 2008). De este modo, el desarrollo tecnológico, las nuevas aplicaciones educacionales y los diferentes estudios sobre los beneficios del uso de dispositivos móviles en el aula, respaldan su aplicabilidad como una herramienta educativa a destacar (Plump y Larosa, 2017; Zichermann y Cunnigham, 2011).

En los últimos años han proliferado las herramientas para gamificar el aprendizaje en el aula y muchas de ellas toman forma de aplicaciones para dispositivos móviles. Una de las más utilizadas es la aplicación Kahoot!, tanto por la sencillez de creación de la experiencia de juego como por su fácil acceso a través de dispositivos móviles. Esta herramienta busca facilitar la evaluación del trabajo, tanto individual como en grupo, y permite conocer de forma inmediata el grado de adquisición de conocimiento de los alumnos e identificar los contenidos que no se han comprendido. Kahoot! se fundamenta en tres valores básicos (jugar, aprender y socializar), que se materializan en una plataforma online y colaborativa en la que, tanto profesores como alumnos, pueden crear cuestionarios interactivos sobre cualquier materia. De esta forma, los estudiantes pueden competir entre sí para saber quién acierta más preguntas en el menor tiempo. La aplicación también permite crear preguntas de opinión para sondear a los alumnos o hacer encuestas sobre algún tema de debate, y puede ayudar a promover la participación de los alumnos a través de las experiencias de aprendizaje activas y colaborativas que plantee el docente.

El presente trabajo tiene un doble objetivo: el primero se centra en analizar las diferentes herramientas de gamificación actualmente disponibles en el mercado para ser utilizadas a través de dispositivos móviles. El segundo objetivo describe una propuesta metodológica de gamificación a través del uso de una de estas herramientas, la aplicación Kahoot!, en los estudios de Ingeniería en la Universitat de Girona. 
Los resultados de esta experiencia de gamificación con Kahoot! muestran como la participación activa de los estudiantes se estimula y aumenta su motivación e interés en la materia. Todo esto deriva en un impacto positivo sobre el índice de asistencia de los alumnos a las sesiones presenciales y al seguimiento general de la asignatura. Además, permite al profesor obtener numerosas evidencias del proceso de aprendizaje de sus alumnos.

\section{El aprendizaje divertido: gamifi- cación}

La adopción de técnicas de Gamificación por parte del profesorado en el aula, el Game Based Learning, GBL, tiene como objetivo optimizar los resultados perseguidos, ya sea para absorber mejor algunos conocimientos, para mejorar alguna habilidad o para recompensar acciones concretas, entre otros (Kapp, 2012).

Así, la neurodidáctica apunta a ciertas características del aprendizaje (Romera, 2018; Willis, 2010) que la Gamificación intenta aprovechar:

\section{- $\quad$ El cerebro aprende cuando se emociona}

A través de la Gamificación se busca explotar esa capacidad de emocionar. Crear algo que llegue a los alumnos, que despierte su interés y les motive a progresar. Una de las principales razones que argumentan los profesores que se animan a gamificar en sus asignaturas es intentar evitar que sus alumnos "desconecten" en sus clases y por tanto se alejen de este objetivo. Muchos docentes afirman que "la falta de motivación de los alumnos es un hecho" y esta afirmación les conduce a culpabilizar a los alumnos de su falta de aprendizaje "si no están motivados, yo no puedo enseñarles" (Fernández, 2015). Así pues, si el docente considera una realidad que los alumnos no están motivados, entonces, antes de intentar transmitir los conocimientos, antes incluso de intentar que el alumno adquiera determinadas competencias y como paso previa a las etapas del aprendizaje por las que pasará el alumno, se debe trabajar la motivación.

Existen muchas teorías de motivación, como la Teoría de la Pirámide de Maslow o la Teoría de los Factores Higiénicos de Hezberg, ambas esenciales para desarrollar un sistema gamificado con sentido y coherencia. Dentro de este sistema existen dos tipos de motivaciones sobre las que actuar: una motivación intrínseca, en la que el usuario realiza determinada actividad por el placer de realizarla, sin que se le otorgue ningún incentivo externo, y una motivación extrínseca que aparece cuando lo que desea el individuo es lo que recibe a cambio de la actividad realizada. Así, el modelo GBL, basado en el juego, se muestra realmente útil ya que consigue motivar a los alumnos, desarrollando un mayor compromiso e incentivando su superación personal.

- El cerebro aprende haciendo, "learning by doing"

Una segunda clave que sustenta la utilización de estas nuevas metodologías es la forma en que aprendemos los seres humanos. Está demostrado por diferentes estudios que con estímulos verbales (clase tradicional/lección magistral) obtenemos un $10 \%$ de tasa de recuerdo a las 72 horas. Es decir, de todo lo que "decimos" en nuestras clases aproximadamente un año después ya no se recuerda casi nada. Pero si a esos mismos alumnos les mostráramos un total de 2.500 imágenes, con una frecuencia de 10 segundos por imagen, la tasa de recuerdo de dichas imágenes a las 72 horas sería del $90 \%$. Y al cabo de un año, todavía recordarían el $63 \%$ de las imágenes visualizadas (Fernández, 2015). En definitiva, cuantas más oportunidades se den de aprender lo visto en el aula mejor.

El "cono de aprendizaje" de Edgar Dale (Dale, 1969) muestra que la mayor tasa de aprendizaje se logrará con un estudiante activo y que se enfrente a simulaciones o situaciones reales. Dale afirmaba que tras 10 días recordamos un $20 \%$ de lo que escuchamos frente al $90 \%$ de lo experimentado (ver Figura 1).
Figura 1 Adaptación del "cono del aprendizaje” de Edgar Dale (Fernández, 2015).

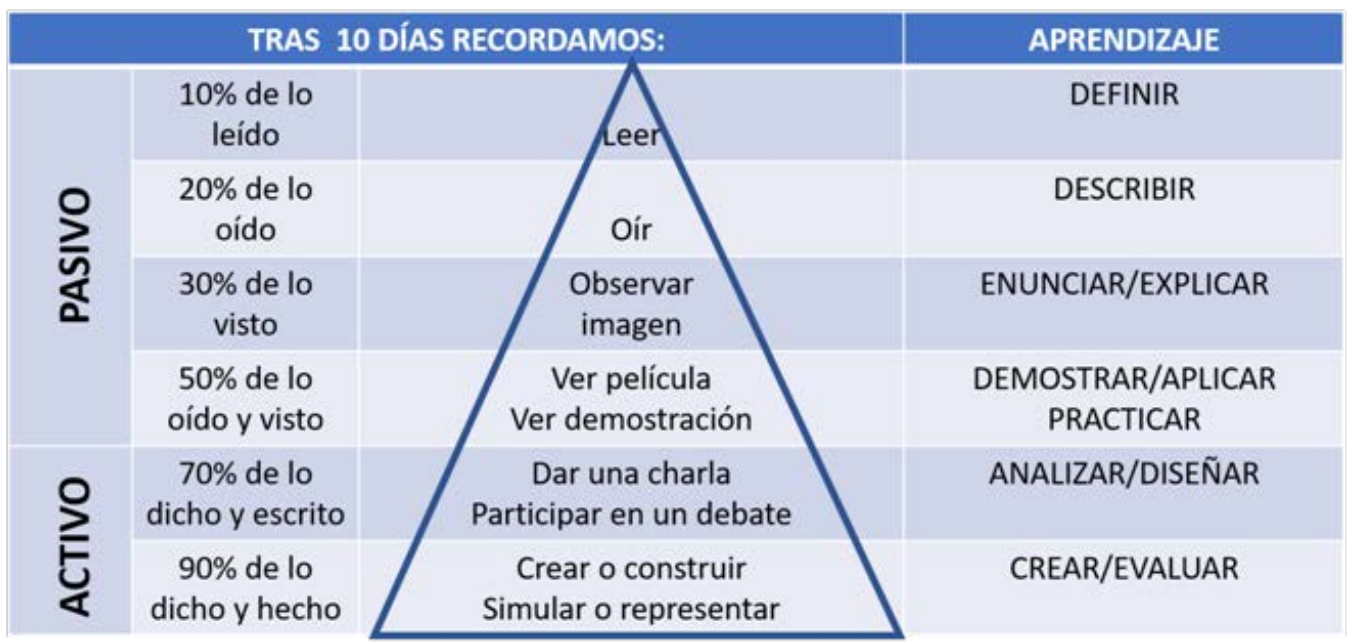


A través de la Gamificación el alumno puede hacer dos cosas esenciales: puede "hacer" (puede experimentar), aunque sea virtualmente, y puede "equivocarse", equivocarse para aprender. Una de las debilidades en el desarrollo de la actividad docente es la incapacidad de dejar a los alumnos equivocarse y volver a empezar. De ese modo únicamente se consigue que tengan miedo a equivocarse. Este hecho es muy importante en ámbitos especialmente sensibles y que vienen utilizando esta metodología de la simulación desde hace décadas, como en el ámbito militar y de la medicina, precisamente donde las consecuencias de los errores humanos son especialmente elevadas. Es importante tener en cuenta que un sistema de enseñanzaaprendizaje correctamente gamificado debe proporcionar un feedback inmediato y continuo.

- El cerebro es un órgano social que mejora el aprendizaje cuando interactúa con otros: en el aula se producen interacciones, eso es innegable, pero con la gamificación podemos modelarlas o conducirlas para que se ajusten al objetivo que persigue el docente

Los mecanismos del juego generan interacción entre los participantes y esto hace que el componente social sea importante, ya sea en el caso de que el objetivo del juego sea competitivo o sea colaborativo (Area et al, 2015). Guillen y Aleson (2012) afirman que la gamificación tiene un efecto positivo sobre la competencia comunicativa y Cortizo et al (2011) consideran que fomenta la participación y genera compromiso. Por su parte Mayo (2007) afirma que mejora la autoconfianza y la cooperación.

El proceso del diseño de una experiencia de gamificación incluye cuatro ingredientes básicos (Werbach y Hunter, 2012):

- El objetivo: Se trata de tener muy claro qué se quiere conseguir con esta experiencia gamificada. Objetivos posibles a perseguir podrían ser el mejorar el clima del aula, el favorecer al aprendizaje de contenidos, el mejorar el rendimiento académico o la promoción del trabajo cooperativo

- La narrativa: Definir de qué irá el juego, cuál será su tema

- La dinámica: Hacen referencia a la motivación del jugador para participar y seguir adelante en la consecución de sus objetivos

- La mecánica: Se trata de definir la metodología concreta del juego que determina cómo se desarrollará en base a parámetros como la puntuación, clasificación o niveles
Las técnicas dinámicas más utilizadas son las siguientes:

- $\quad$ Recompensa: obtener un premio merecido

- $\quad$ Estatus: situarse en un nivel jerárquico social valorado

- Logro: superación o satisfacción personal

- Competición: afán de competir e intentar ser mejor que el resto.

En función de la dinámica que se busque, se deberá utilizar unas técnicas mecánicas más que otras. Estas técnicas mecánicas, extrapoladas de los juegos, se basan en el modo de recompensar a los alumnos en función de los objetivos alcanzados (Ramírez, 2014). Algunas de ellas son las siguientes:

- Acumula puntos: se asigna un valor cuantitativo a determinadas acciones y se van acumulando a medida que se realizan

- $\quad$ Niveles: se definen una serie de niveles que el usuario debe ir superando para llegar al siguiente

- Premios: a medida que se consiguen diferentes objetivos se van entregando premios a modo de "colección"

- Regalos: bienes que se dan al jugador o jugadores de forma gratuita al conseguir un objetivo

- Clasificación: clasificar a los usuarios en función de puntos u objetivos logrados, destacando los mejores en una lista o ranking

- Desafíos: competiciones entre los usuarios, el mejor obtiene los puntos o el premio

- Retos: conseguir resolver o superar un reto u objetivo planteado, ya sea solo o en equipo

Pero la adopción del GBL también tiene algunos puntos débiles que se deben tener en cuenta. Un primer aspecto a considerar es el económico. Este nuevo marco de aprendizaje está comenzando a convertirse en un negocio muy lucrativo y hay que explorar bien todas las alternativas si a opción es adquirir una herramienta en el mercado de "juegos de aprendizaje”. Expertos del sector predicen que este mercado crecerá un $500 \%$ en los próximos años pudiendo llegar a alcanzar más de 11billones de dólares en 2020 (Polites, 2017). Repositorios como el The Gamification Repository creado por el grupo de investigación INSISOC de la Universidad de Valladolid o el Lean Simulations creado por ingeniero canadiense Martin Boersema permiten al docente tener una amplia visión de las posibilidades existentes en el campo de la gamificación dentro del ámbito de la docencia en los estudios de Ingeniería. 
Otro aspecto problemático es reducir la gamificación a la mera consecución de premios. Existe el peligro que el estudiante acabe actuando en base a una rápida y automática realización de las tareas e impida la correcta asimilación del aprendizaje, y por tanto deje en segundo término el objetivo planteado. Las ilusión ganas de obtener premios, insignias u otro tipo de regalos inmateriales no perviven para siempre y aunque puede motivar al principio, puede pasar que transcurrido un tiempo ya no sean efectivas. Es muy complicado y laborioso encontrar el término medio en un juego para asegurar que, dentro de un entorno interesante, puedan realizar un aprendizaje efectivo asumiendo los objetivos que se persiguen.

Finalmente otro punto débil a considerar es que la mayoría de juegos educativos comercializados bajo la denominación "gamificación", son lineales y pueden no ajustarse a una educación personalizada, donde los diferentes niveles, intereses y estilos de aprendizaje no cuadren con los objetivos del docente.

Así pues, para gamificar el aula se necesita preparación, tiempo, dinero y algo muy importante: una adecuada formación del profesorado.

\section{Gamificación con dispositivos móviles}

La adopción de la tecnología digital en el ámbito de la educación no es ninguna moda pasajera, sino que es un cambio que ha llegado para permanecer y que está transformando completamente muchos ámbitos de la sociedad. Así, en el informe The NMC Horizon Report 2013 Higher Education Edition, que identifica las tendencias en la adopción de tecnologías en la educación superior, ya se destacaban la Gamificación y las Apps (o aplicaciones móviles) como aspectos susceptibles de tener un importante impacto en el modelo de enseñanzaaprendizaje en educación superior en un horizonte temporal de 1 a 3 años. Posteriormente, en el informe del 2015 aparece el concepto de BYOD, Bring your own device, como tendencia a adoptar a corto plazo. El BYOD es un modelo tecnológico en el cual los estudiantes llevan su dispositivo personal al aula con el objeto de aprender (Alberta, 2012).

Así pues, la utilización de los dispositivos móviles, ya sean teléfonos o tablets, en el aula es una práctica cada vez más habitual en la enseñanza superior (Alejandre, 2017; González y Salcines, 2015). Esta, debe diferenciarse del mobile learning, concepto asociado al conjunto de métodos de enseñanza-aprendizaje que se basa en recepción o entrega de información para ser aplicados en cualquier momento y lugar a través, exclusivamente, de tecnología móvil y que se lleva a cabo en diferentes contextos, cuyo objetivo es apoyar otros medios de enseñanza con el fin de lograr un aprendizaje auténtico (Ramos et al, 2010). Es decir, éste articulo contempla el uso del móvil como una herramienta más dentro del aula como pueden ser los ordenadores, las tablets o los libros.
Diferentes autores afirman que la introducción de dispositivos móviles en el proceso de enseñanzaaprendizaje abre un amplio abanico de potencialidades educacionales, algunas de ellas aun por explorar (Navaridas, 2013). Entre los beneficios que se asocian habitualmente a la utilización en el aula de este tipo de dispositivos móviles (Guerrero et al, 2016; Morris y Chikwa, 2013; Moya et al, 2016; Roig et al, 2016; Villegas, 2016; Zarzycka, 2014) se destacan los siguientes:

- $\quad$ Flexibilidad en el uso a través de la portabilidad y la conectividad en cualquier lugar y momento, sin necesidad de disponer de aula informática

- Enfatizan en la competencia digital

- $\quad$ Permiten utilizar herramientas educativas tecnológicas (trabajar con realidad aumentada, códigos QR, juegos con mecánica tipo concurso o quiz, uso de aplicaciones específicas por materias o competencias, etc.)

- Favorecen el seguimiento del método enseñanza-aprendizaje, facilitando el proceso de evaluación continua

- Aumentan la motivación y participación de los alumnos a través de las experiencias de aprendizaje activas y colaborativas que estos dispositivos permiten

Así, a través de aplicaciones para móviles y tablets se puede acceder a herramientas y recursos que sin duda ayudarán al docente a diseñar mejores experiencias de gamificación en el aula, pero siempre teniendo en cuenta el reto de cómo introducir esta herramienta con un sentido, es decir, dentro de una programa docente y en el marco de una propuesta metodológica.

Tourón et al (2014) apunta a tres aspectos fundamentales en los que el uso de la tecnología móvil en el aula beneficia a los estudiantes:

- Motivación: los alumnos aumentan el interés en la actividad a realizar, mejorando su actuación en un ambiente de aprendizaje positivo.

- Habilidades sociales: los alumnos, como miembros de un grupo, refuerzan sus habilidades sociales, mejoran su espíritu de grupo, comunicación interpersonal y empatía, creciendo en responsabilidad y compromiso personal.

- Habilidades cognitivas: los alumnos desarrollan su creatividad generando nuevas ideas o contenidos, mejorando los procesos de adquisición de la información y promoviendo su capacidad analítica, de síntesis y evaluación de esta información. 
Las llamadas Aulas 3.0 empiezan a ser una realidad, pero en muchas ocasiones no se hace un uso del potencial que tienen y la gamificación a través de dispositivos móviles es una forma de evolucionar hacia el concepto de Educación 3.0 (Gerstein, 2014; Romera, 2018).

Un primer objetivo de este trabajo se centra en analizar las diferentes herramientas de gamificación disponibles en el mercado para ser utilizadas a través de dispositivos móviles. Actualmente existen multitud de herramientas para gamificar el aprendizaje en el aula y muchas de ellas toman forma de aplicaciones para tablets y smartphones (Educación 3.0, 2017; Educación 3.0, 2018). En este trabajo se han revisado las herramientas más referenciadas en artículos, revistas especializadas, blogs y webs sobre educación e innovación docente y se ha propuesto la siguiente clasificación (ver Figura 2), según sus objetivos educacionales o ámbito de utilidad:

- Plataformas de gestión del aula: son aplicaciones que ayudan al docente a administrar un aula en base a puntuaciones sobre el desarrollo de las actividades que se proponen, el comportamiento e interacciones con compañeros en el aula, entre otros aspectos (Arjona y Gamiz, 2013). (ClassDojo, Toovari, Classkick, Edmodo, Moodle, Google Classroom, SWAD)

- Videojuegos: en los que el alumno tiene que ir resolviendo ciertos retos y problemas para ir avanzando y mejorar. Deben cooperar y participar en misiones para ir ganando puntos con el objetivo de mejorar su equipo. El objetivo, ir avanzando de forma colaborativa a la vez que aprenden y desarrollan su conocimiento (Codecombat, Classcraft, Minecraft, Zondle)

- Unidades de estudio o Flaschcards: una unidad de estudio es una lista de términos con sus definiciones correspondientes (o preguntas con sus respuestas) que se pueden estudiar con los distintos modos de estudio de las aplicaciones. Las unidades de estudio pueden incluir palabras, imágenes, diagramas y audio texto-hablado (Quizlet, Brainscape)

- Contenidos en línea: este tipo de aplicaciones permiten la creación de presentaciones en línea y compartirlas a los estudiantes en tiempo real. Mientras el docente desarrolla el tema, los estudiantes pueden ir recibiendo contenido en sus dispositivos, como por ejemplo preguntas, imágenes o cualquier tipo de material de apoyo. $\mathrm{Su}$ objetivo es convertir las clases en bidireccionales para aprovechar las explicaciones al máximo. Algunas permiten enviar preguntas, lecciones y todo tipo de material a los estudiantes para que estos, a través de sus dispositivos móviles, puedan resolverlos al instante. Lo mejor de todo es que el profesor ve en tiempo real las respuestas de cada uno de sus alumnos, lo que le permite evaluarlos inmediatamente (Pear Deck, Nearpod, Formative, Edulastic)

- $\quad$ Edición de vídeos educacionales: este tipo de aplicaciones permiten crear vídeos y añadir texto o comentarios a ellos, también permiten evaluar al alumnado de una forma sencilla. Se añaden ciertas preguntas a lo largo del vídeo y la plataforma registra las respuestas de cada alumno para que luego el docente pueda determinar su nivel de comprensión respecto de la materia (EDPuzzle, PlayPosit)

- $\quad$ Concursos de preguntas y respuestas (Quiz): permiten recrear en el aula el típico ambiente de los concursos de preguntas y respuestas donde el profesor toma el rol del presentador del concurso y los alumnos son los concursantes, que pueden participar e modo individual o en grupo. Algunas variantes permiten jugar al popular juego Trivial y ofrecen la posibilidad de configurar un juego de Trivial propio con los alumnos de manera colaborativa (Socrative, Trivinet, Quizziz, Plickers, Kahoot!, Google Forms, Flipquiz, Mentimeter, Quizalize, Triventry)
Figura 2 Herramientas de gamificación a través de dispositivos móviles

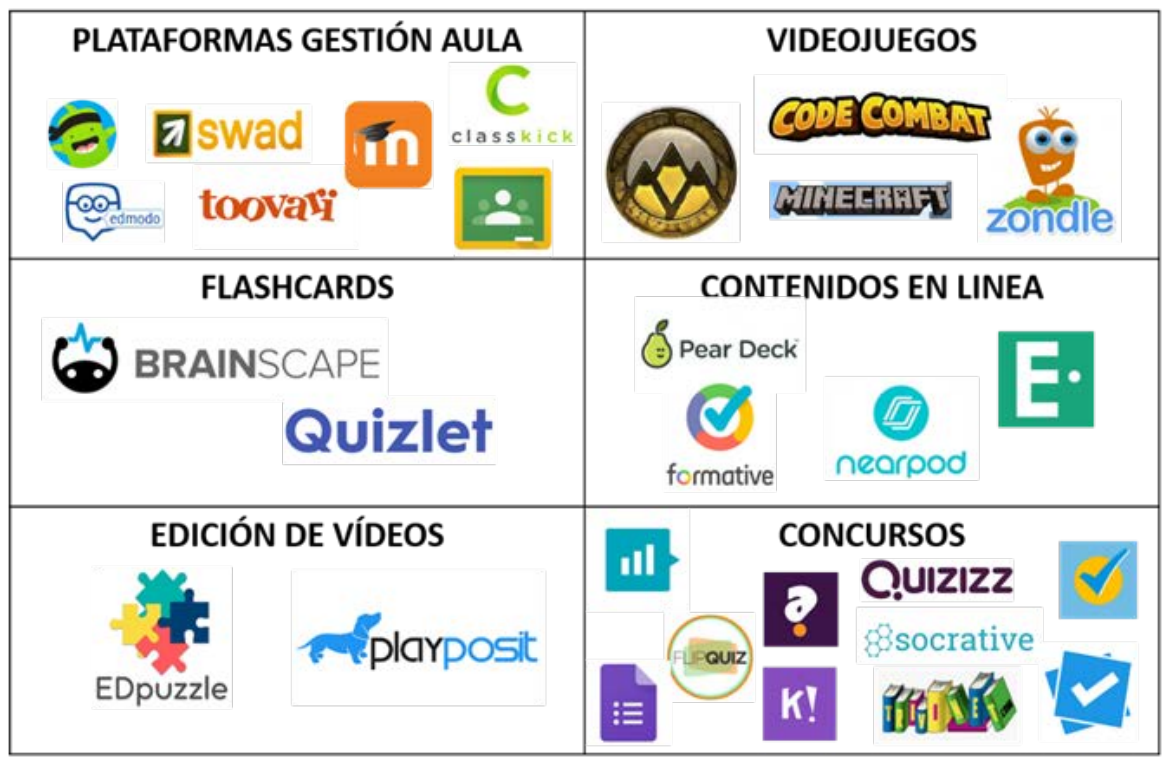




\section{La experiencia de Kahoot!}

En la revisión de aplicaciones para gamificar el aula a través de dispositivos móviles realizada en el apartado anterior se demuestra que las más numerosas son las de tipología de concursos de preguntas y respuestas; y es en esta categoría en la que se enmarca la propuesta metodológica que se presenta a continuación.

Se ha escogido Kahoot! por ser una de las plataformas gratuitas de Gamificación más conocidas a nivel mundial y actualmente es la aplicación de preguntas y respuestas más usada en la comunidad educativa, con 33 millones de usuarios activos y 3,5 millones de profesores de 180 países (Iglesias, 2016). También se ha escogido por su simplicidad en el uso y porque Kahoot! se muestra como una herramienta muy bien valorada por estudiantes de educación superior (Plump y Larosa, 2017; Sellés et al, 2016).

Kahoot! es una herramienta basada en el sistema GSRS (Game-based Student Response System) que buscan facilitar la evaluación del trabajo tanto individual como en grupo y permiten conocer de forma inmediata el grado de adquisición de conocimiento de los alumnos e identificar los contenidos que no se han comprendido (Fies y Marshall, 2006). La clave de esta aplicación GSRS es que, en base al análisis de los resultados en clase, permite el feedback instantáneo con los alumnos. Los datos generados por la aplicación también permiten conocer la evolución de cada estudiante y por tanto facilitar su atención personalizada y su evaluación de manera continuada, ya sea formativa o sumativa.
Kahoot! fue creada en 2013 por el profesor Alf Inge Wang de la Norwegian University of Science and Tech en colaboración con otros profesionales (TICBEAT, 2016) y se basa en tres valores básicos (jugar, aprender y socializar), que se hacen realidad en una plataforma online y colaborativa en la que, tanto docentes como estudiantes, tienen la posibilidad de crear cuestionarios interactivos sobre cualquier materia. De este modo, los alumnos pueden competir entre sí o en grupo de una manera lúdica y sencilla.

\subsection{Funcionamiento de Kahoot!}

La plataforma Kahoot! está formada por dos páginas web. La primera es la llamada getKahoot! que es la que utiliza el profesor para poner en marcha un Kahoot!, utilizar los de otros o crear los suyos propios. La otra es Kahoot.it! que es la que utiliza el alumno para entrar en cada "partida”.

Una de las ventajas de Kahoot! es que los alumnos pueden entrar sin necesidad de descargarse la aplicación, simplemente lo hacen con un PIN de entrada, que les facilitará el profesor. Con un dispositivo móvil el alumno entra en el Kahoot! y se identifica con un nickname. El juego se visualiza en la pantalla del aula a través del proyector y empieza cuando todos los participantes se ven en pantalla. En este momento el profesor lanza el juego. En la pantalla aparece la pregunta o imagen sobre la que contestar y los participantes deben contestar pulsando a través de su dispositivo móvil en el color de la respuesta correcta. Una vez se agota el tiempo de respuesta todos los alumnos ven la respuesta correcta (feedback inmediato) y las opciones que han escogido cada uno de los participantes (ver Figura 3).
Figura 3 Visualización de Kahoot!

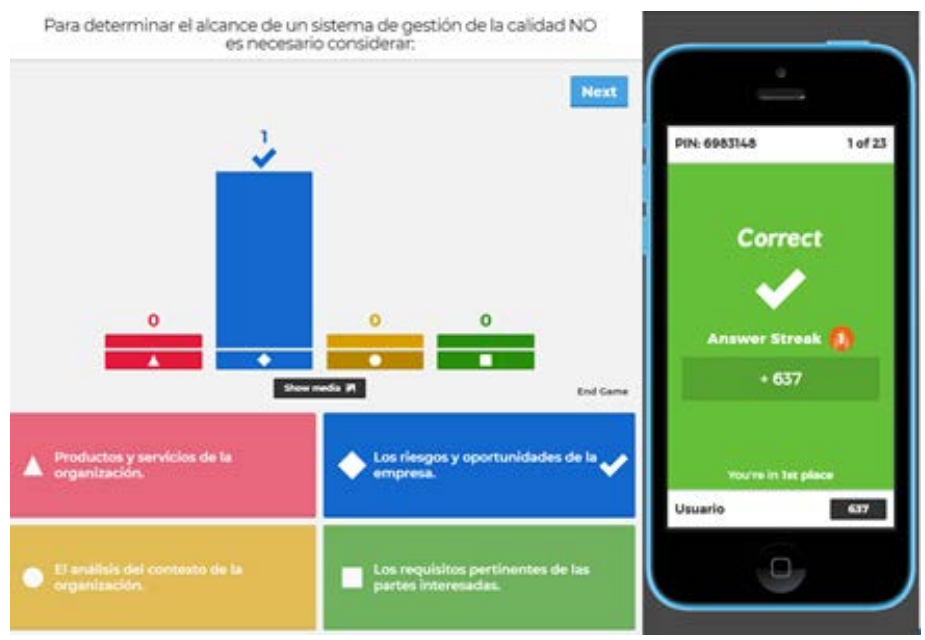


En este momento, en función del acierto y la rapidez de respuesta, la aplicación les otorga puntos. En este momento el ranking de los alumnos se muestra en pantalla donde aparecen únicamente los cinco mejores clasificados, lo que motiva enormemente a los estudiantes. Este proceso se repite para cada una de las preguntas planteadas y al final, el profesor recibe un archivo Excel con todas las respuestas de los alumnos participantes que puede compartir con ellos para realizar sus análisis y posterior seguimiento de la asignatura por parte de los alumnos o clarificar inmediatamente conceptos que quizás no han quedado claros. El alumno puede volver a realizar el Kahoot! las veces que desee en un proceso de aprendizaje por prueba y error.

Los pasos que debe seguir el docente para utilizar la plataforma y crear sus Kahoot! son muy sencillos (Espeso, 2018). El primer paso es registrarse en Kahoot!. Una vez se activa la cuenta del docente, éste ya puede autentificarse en la plataforma para acceder a la web. Allí se pueden buscar Kahoot!s creados por otros usuarios e iniciarlos, o crear uno propio desde cero, a través de las cuatro opciones (Quiz, Jumble, Discussion, Survey). Se puede crear un Kahoot! en base al típico juego de preguntas y respuestas (Quiz) pero también existen otras tres opciones (discusión, encuesta, mezcla) que pueden ser muy interesantes para usar en clase. Partiendo de la base que se escoge la opción Quiz, se abre la página para crearlo. Se deben de rellenar los campos: título, descripción, idioma, público objetivo, etc. que serán de utilidad cuando se realicen búsquedas de Kahoot! públicos. Al empezar a crear las preguntas, la plataforma pide algunos datos, con una interfaz intuitiva y fácil de utilizar, que se deberán repetir para cada pregunta que se cree: límite de tiempo (por defecto 20 segundos), recursos adicionales (en el caso en el que se quiera ampliar la información de la pregunta o media), posibilidad de añadir una imagen o un vídeo para contextualizar la pregunta, o también para plantear preguntas alrededor de la imagen o vídeo que se haya insertado. El Kahoot! creado se guarda en la sección "My Kahoot!s" de la página principal, y se puede acceder a ellos y jugarlos cuando se quiera. Éste, puede ser sobre una lección o tema específico, o sobre una material al completo. Incluso, también puede plantearse como una actividad transversal en el ámbito del aprendizaje por proyectos, utilizando preguntas sobre más de una asignatura. En referencia a cuál es el número de preguntas idóneo, depende mucho tanto del tema como del tipo y número de los alumnos, aunque se recomienda empezar con 10 preguntas y plantear alrededor de un máximo de 20. También es aconsejable utilizar los recursos multimedia que Kahoot! que permiten insertar imágenes y gráficas. Estas pueden ser accesorias o ser la base para realizar las preguntas. Además de crear tus propios Kahoot!s, una de las opciones más interesantes de esta plataforma es que cualquiera puede compartir sus creaciones con otros usuarios. Es posible acceder a una biblioteca de Kahoot!s (a través de la sección "Discover") y buscar, a partir de palabras clave, Kahoot!s creados por profesores de todo el mundo. Esto requiere que estén bien descritos y definidos, añadiendo información sobre el estudio, curso y materia a la que aplica.

\subsection{Propuesta metodológica}

A continuación se presenta una propuesta metodológica de Gamificación, a través del uso de Kahoot!, que se ha implementado en el curso 2017-2018 por primera vez en una asignatura de los estudios de Ingeniería en la Universitat de Girona, la asignatura de Organización y Gestión de Empresas, asignatura obligatoria de segundo curso, compartida por 4 grados: Ingeniería mecánica, Ingeniería electrónica industrial y automática, Ingeniería Eléctrica e Ingeniería Química.

La propuesta metodológica que se presenta (Figura 4) se fundamenta en 7 pasos:

1) Clase Magistral (1h): definido por parte del profesor el tema o contenido del curso que se quiere trabajar, este realiza inicialmente una clase magistral donde se presentan los contenidos básicos y suficientes para que los estudiantes puedan comenzar a desarrollarlos.

2) Kahoot! \#1 (0,5h): Posteriormente, y para establecer los nuevos conocimientos y verificar que el inicio del aprendizaje ha sido el correcto, se realiza un primer Kahoot! en el aula en el que se presentarán los conceptos teóricos trabajados en la clase magistral, lo que permite al profesor comprobar el nivel de aprendizaje adquirido por sus alumnos en tiempo real. Este Kahoot! inicial lo juegan en grupos de dos alumnos para favorecer el debate y la cooperación entre iguales. Para ello se da un tiempo de resolución de la pregunta suficientemente amplio para favorecer la discusión entre los integrantes del grupo (60 segundos). Kahoot! permite fijar un tiempo de respuesta distinto para cada pregunta entre las siguientes opciones (5, 10, 20, 30, 60, 90 o 120 segundos). En este Kahoot! inicial se plantean entre $10-12$ preguntas. 
Figura 4 Modelo de

propuesta metódológica de

gamificación

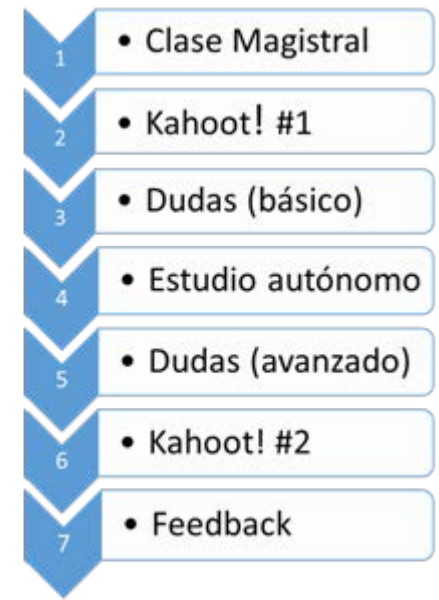

3) Resolución de dudas-nivel básico (0,5h): Una vez que se han completado estos pasos, es necesario superar las posibles desviaciones surgidas en el proceso de enseñanza-aprendizaje. Para ello se requiere una reflexión en el aula, para que todas las dudas puedan ser resueltas y los contenidos se afiancen correctamente.

4) Estudio autónomo (6h): Una vez establecidos los contenidos teóricos básicos, los estudiantes deben continuar desarrollando su propio aprendizaje de manera autónoma para afianzarlos y profundizarlos, a través de actividades planteadas por el profesor. Para ello se utilizaran diferentes alternativas en función de los contenidos a trabajar en cada momento (visionado de videos TEDTalk u otros contenidos facilitados por el profesor). En ambos casos el alumno realiza la actividad de manera autónoma en casa y posteriormente contesta a un cuestionario de autocorrección online a través de Moodle. Otra opción es la resolución de ejercicios de autocorrección que el alumno debe realizar a través de la plataforma ACME (Rafart et al, 2017). En todos los casos el profesor puede controlar los intentos y los resultados de los ejercicios y análisis de las respuestas obtenidas. En esta propuesta metodológica, se plantea la realización de 3 actividades, todas ellas se realizan de manera autónoma fuera del aula y tienen una evaluación formativa, donde la "nota” obtenida no es importante y no se tiene en cuenta en la evaluación final de la asignatura, sino que lo importante es que el alumno puede hacer las dos cosas esenciales del aprendizaje, "hacer”, aunque sea virtualmente, y “equivocarse”, equivocarse para aprender.

5) Resolución de dudas-nivel avanzado (1h): Posteriormente a la realización de cada una de las actividades llevadas a cabo de manera autónoma por el alumno fuera del aula, dentro del aula el profesor plantea una revisión de los contenidos tratados y se clarifican las dudas que hayan surgido durante la realización de las actividades. El profesor puede llevar preparada esta sesión de clarificación de dudas en base a los resultados de la actividad realizada por parte de los alumnos, ya que él ha podido consultar y seguir la evolución del trabajo de sus alumnos en todo momento y puede incidir en los conceptos que considere más importante clarificar por su dificultad.

6) Kahoot! \#2 (0,5h): La propuesta metodológica finaliza con la realización en el aula de un nuevo Kahoot! sobre los contenidos trabajados. Este segundo Kahoot! puede contener preguntas muy similares a las del Kahoot! inicial y otras, un poco más complejas, que permitan visualizar el trabajo del alumno, que debe de haber profundizado en los contenidos planteados con su trabajo autónomo y otras posibles actividades realizadas en el aula con profesor. Este segundo Kahoot! se realiza de manera individual, fomentando en este momento la competición entre iguales y no la cooperación, como se buscaba en el Kahoot! inicial. En este caso se plantean 20 preguntas, con un tiempo de respuesta de 20 segundos.

7) Resolución de dudas-feedback final (0,5h): Este paso acabo con el feedback final del profesor hacia los alumnos en relación a los posibles aspectos problemáticos que haya podido identificar durante la resolución de este segundo Kahoot!, fomentando a la vez la resolución de las dudas que aún puedan existir entre los alumnos participantes.

La metodología que se presenta ha sido diseñada considerando al estudiante como un elemento activo en la construcción de su proceso de aprendizaje.

Los recursos necesarios para implementar esta metodología son, por parte del centro docente, los que habitualmente se encuentran en todas las universidades y se reducen a una pizarra digital o proyector y un ordenador con conexión a internet. Por parte del alumno este debe disponer de un dispositivo móvil (smartphone o tablet) con acceso a internet o a la Wi-Fi del centro. 
Las estrategias didácticas y metodológicas utilizadas en el diseño de esta propuesta, se han definido para poder ser implementadas en cuatro sesiones presenciales de una hora de duración cada una de ellas, más seis horas de trabajo autónomo por parte del alumno en la resolución de las tres actividades de autoevaluación planteadas. En definitiva, aplicar esta propuesta metodológica, implica una dedicación mínima por parte del alumno de 10 horas. Por parte del profesor, la dedicación requerida es mucho mayor e incluye el diseño de los dos Kahoot!, el planteamiento de las tres actividades para el estudio autónomo y el seguimiento posterior de los alumnos en su desarrollo, y la preparación de la sesión de resolución de dudas (nivel avanzado). En este caso, se ha aplicado esta metodología en cinco ocasiones a lo largo del semestre, con el objetivo de mejorar el rendimiento de los alumnos en aquellos contenidos más complejos de asimilar por parte del alumno.

Finalmente, también se deben considerar los aspectos asociados al sistema de evaluación. En la literatura se definen dos grandes tipos básicos de evaluación en el proceso evaluativo del alumno: la evaluación sumativa y la evaluación formativa o continua (Rosales, 2014). La evaluación sumativa tiene por objetivo establecer balances fiables de los resultados obtenidos al final de un proceso de enseñanza-aprendizaje. Esta evaluación pone el acento en la recogida de información y en la elaboración de instrumentos que posibiliten medidas fiables de los conocimientos a evaluar. Tiene por finalidad la asignación de una nota o cualificación a las diferentes actividades de evaluación que permiten determinar si el estudiante supera o no la actividad planteada. El segundo tipo de evaluación es la evaluación formativa o continuada. Esta se realiza durante el desarrollo del proceso de enseñanza-aprendizaje para localizar las deficiencias cuando aún se está en posibilidad de remediarlas, esto es, introducir sobre la marcha rectificaciones a que hubiere lugar en el proyecto educativo y tomar las decisiones pertinentes, adecuadas para optimizar el proceso de logro del éxito por el alumno (Rosales, 2014). En este caso el objetivo principal es determinar el grado de adquisición de competencias e identificar aquellas que se deben trabajar de forma más exhaustiva, es decir funciona para revisar el progreso de los estudiantes y a considerar las opciones de aprendizaje adicional para asegurar el éxito del aprendizaje (Margalef, 2014). Así, esta propuesta metodológica se basa en la evaluación formativa ya que lo que se pretende conseguir es que el alumno pueda "hacer" y “equivocarse”, sin miedo a la "nota” obtenida. Por tanto, no se tiene en cuenta ninguna calificación en la evaluación final de la asignatura, sino que lo importante es potenciar la motivación del alumno en el proceso de enseñanza-aprendizaje con el objetivo de mejorar su capacidad de comprensión de los contenidos trabajados a la vez que detectar las posibles deficiencias. En este sentido, cabe destacar que la participación en esta experiencia no era obligatoria para los alumnos, aunque la mayoría han participado en todas las ocasiones en las que se ha planteado.

\section{Resultados y discusión}

En este apartado se presentan los resultados de la aplicación de la metodología docente desarrollada en el apartado anterior. Se han recogido datos en referencia a varios indicadores con el objetivo de evaluar el grado de compromiso, motivación y rendimiento de los alumnos frente a la experiencia implementada por primera vez en el curso 2017-2018. Estos indicadores han sido utilizados previamente en otros estudios (Barata et al, 2013; Mandernach et al, 2011).

El periodo temporal del estudio de estos indicadores comprende cuatro cursos académicos $\mathrm{y}$, en todos ellos, la asignatura ha sido impartida siempre por el mismo docente. Se han analizado parámetros como, el número de accesos al Moodle de la asignatura donde el alumno puede consultar todos los recursos de la asignatura facilitados por el profesor, el número de mensajes enviados al profesor planteando consultas, el número de tutorías presenciales realizadas, el grado medio de asistencia a clase, el número de alumnos no presentados y la nota final media obtenida. Los datos obtenidos del curso 1718 se han comparado con los obtenidos en los tres cursos anteriores en los que no se aplicaba la metodología descrita. La Tabla 1 recoge toda esta información.

El indicador "Accesos al Moodle" se ha calculado considerando el número total de accesos al Moodle de la asignatura, entre el 1 de septiembre y el 31 de julio del curso académico en cuestión. La evolución de este indicador muestra un aumento muy significativo en el curso 17-18 respecto a los cursos anteriores, pasando de una mediana de 87 accesos por alumno en el curso 16-17 a los 154 del curso $17-18$, lo que significa un incremento de los accesos del $77 \%$. Este gran aumento en el acceso y consulta general de los contenidos del curso sugiere una importante mejora en el interés y entusiasmo de los alumnos atribuible a esta experiencia de aprendizaje gamificada. 
Tabla 1 Evolución de los indicadores

\begin{tabular}{|c|c|c|c|c|}
\hline & $17-18$ & $16-17$ & $15-16$ & 14-15 \\
\hline Alumnos matriculados & 78 & 83 & 92 & 85 \\
\hline Accesos a Moodle & 12008 & 7221 & 6624 & 5421 \\
\hline Accesos a Moodle / Alumnos & 154 & 87 & 72 & 64 \\
\hline Mensajes & 112 & 20 & 15 & 6 \\
\hline Mensajes / Alumnos & 1,43 & 0,24 & 0,16 & 0,07 \\
\hline Tutorías & 18 & 4 & 6 & 8 \\
\hline Tutorías / Alumnos & 0,23 & 0,05 & 0,07 & 0,09 \\
\hline Asistencia media a clase & 65 & 49 & 58 & 64 \\
\hline Asistencia media a clase (\%) & $83 \%$ & $59 \%$ & $63 \%$ & $65 \%$ \\
\hline Alumnos NP (\%) & $6 \%$ & $5 \%$ & $5 \%$ & $8 \%$ \\
\hline Nota final asignatura (sobre 10) & 6,4 & 5,9 & 6,7 & 6,3 \\
\hline
\end{tabular}

El número de mensajes enviados por los alumnos al profesor por correo electrónico ha experimentado una evolución a la alza aún más espectacular que el anterior indicador, pasando de 20 mensajes recibidos en el curso 16-17 (0,24 mensajes por alumno) a los 112 del curso 17-18 (1,43 mensajes por alumno) lo que significa un incremento de casi el 496\%. En cuanto a las tutorías presenciales solicitadas por los alumnos al profesor, estas también han aumentado, pasando de las 4-5 de los cursos anteriores, que representaban alrededor de 0,07 tutorías por alumno, a las 18 del curso 17-18 que significan 1,43 tutorías por alumno. La evolución de estos dos indicadores sugiere, no solo una mayor motivación por parte del alumno, sino también un mayor grado de compromiso y proactividad para aclarar conceptos y resolver dudas.

La asistencia media a clase se ha calculado como el cociente entre la media de la asistencia diaria en el curso académico y el número de alumnos matriculados en ese curso. Este indicador muestra también avances notables respecto a los tres cursos anteriores, donde su evolución era precisamente a la baja. Esta evolución reafirma el importante cambio logrado en el interés por parte de los alumnos a asistir a clase y es muy alentadora para continuar trabajando en esta línea ya que los alumnos, no solo han asistido más a clase sino que han manifestado una mayor disposición a participar en discusiones y debates de una manera voluntaria y proactiva.

El número de alumnos no presentados al examen final y que han abandonado la asignatura no ha sufrido cambios destacables en los años analizados situándose alrededor del $5 \%$ del total de alumnos matriculados. Tampoco lo ha hecho el indicador de "Nota final asignatura", calculado como el rendimiento académico de los alumnos en base a la nota final que han obtenido de la asignatura. Vemos que esta se sitúa dentro de los parámetros habituales de la asignatura (6,3 de promedio sobre 10) y no ha experimentado cambios significativos. Cabe destacar en este caso que, el sistema de evaluación de la asignatura no se ha modificado en los años analizados y que el docente ha sido el mismo por lo que los datos obtenidos no se han podido ver afectados por posibles cambios atribuibles a estos aspectos.

Una vez finalizado el curso 17-18, también se ha recogido mediante un cuestionario la valoración cualitativa de los estudiantes sobre esta experiencia de gamificación del aula. En la Tabla 2 se indica el porcentaje de respuesta obtenido según el grado al que pertenece el alumno. 
Tabla 2 Población y respuesta según grado

\begin{tabular}{lcccc}
\hline & GEM & GEQ & GEE & GEEIA \\
\hline Matriculados & 38 & 12 & 12 & 16 \\
\hline Respuestas & 20 & & & \\
\hline \% Respuestas & & 9 & & 12 \\
\hline
\end{tabular}

En el cuestionario se les ha pedido que califiquen una serie de afirmaciones, utilizando una escala de Likert de cinco puntos, donde 1 significa "totalmente en desacuerdo" y 5 “totalmente de acuerdo". Los resultados obtenidos se desarrollan a continuación y se recogen en la Tabla 3.

Los alumnos han considerado que la experiencia ha funcionado muy bien (4) y, preguntados en particular por la herramienta Kahoot!, han manifestado que es muy sencilla de utilizar (5). Al comparar con otros cursos, han considerado que el curso ha sido mucho más motivador (5) e interesante (4) que el resto de los que han cursado. También han manifestado que la metodología propuesta les ha obligado a estudiar más y de manera más continuada en casa antes de asistir a clase (4) y que obtener una puntuación más elevada que los compañeros y aparecer en la lista de los cinco mejores clasificados del Kahoot! es lo que les ha motivado más a estudiar (4). Los alumnos han considerado que el curso ha requerido de más trabajo (4) pero que la experiencia ha contribuido positivamente a su aprendizaje (4) y que la gamificación debería extenderse a otros cursos (5). Los alumnos también han valorado muy positivamente (5) que los Kahoot! y las actividades propuestas no tuvieran una evaluación sumativa y les permitiera "hacer" y "equivocarse" para "aprender". Los alumnos también han expresado que esta experiencia les ha permitido interactuar más con el resto de compañeros en el aula y aumentar la socialización del grupo (5).

Otros aspectos de la experiencia gamificada sobre los que se ha preguntado a los alumnos no han sido tan favorables. Así, se han quejado en algunos casos de que problemas puntuales de conexión a internet les ha causado problemas en la participación en los Kahoot! (3). También han surgido algunas opiniones no tan favorables en cuanto a la limitación del tiempo de respuesta establecido por el profesor (3) y en la limitación del número de caracteres que se pueden utilizar para plantear las cuestiones y redactar las posibles respuestas, y que los alumnos consideran que algunas veces pueden llevar a alguna la confusión por su brevedad (3).
Tabla 3 Valoración cualitativa de la experiencia metodológica

\begin{tabular}{|c|c|c|c|c|c|}
\hline PREGUNTAS DEL CUESTIONARIO & MEDIA & GEM & GEQ & GEE & GEEIA \\
\hline La experiencia metodológica ha funcionado muy bien & 4 & 5 & 4 & 3 & 4 \\
\hline Kahoot! es muy sencillo de utilizar & 5 & 5 & 5 & 5 & 5 \\
\hline Mi motivación ante esta asignatura es superior al resto & 5 & 5 & 5 & 5 & 5 \\
\hline Mi motivación se basa en que quiero aparecer en el Top 5 de Kahoot! & 4 & 5 & 5 & 3 & 3 \\
\hline La experiencia metodológica hace la asignatura más interesante & 4 & 5 & 4 & 3 & 4 \\
\hline La experiencia ha hecho que estudie más y de modo más continuado & 4 & 4 & 4 & 4 & 4 \\
\hline La experiencia ha contribuido positivamente a mi aprendizaje & 4 & 4 & 4 & 4 & 4 \\
\hline La gamificación debería extenderse a otras asignaturas & 5 & 5 & 5 & 5 & 5 \\
\hline Valoro positivamente que la experiencia sea de evaluación "formativa" & 5 & 3 & 4 & 4 & 5 \\
\hline La experiencia ha contribuido a la socialización del grupo & 5 & 5 & 5 & 5 & 5 \\
\hline He tenido problemas puntuales de conexión en los Kahoot & 3 & 2 & 3 & 3 & 4 \\
\hline El redactado de las preguntas a veces es demasiado breve & 3 & 3 & 3 & 3 & 3 \\
\hline
\end{tabular}


Para finalizar, se realizan una serie de recomendaciones que se desprenden de esta experiencia gamificada. Se considera que la temporización de los Kahoot planteados es clave para el buen desarrollo de los mismos. Así, se recomienda que estos no consten de demasiadas cuestiones y se aconseja no superar los 30 segundos de tiempo para resolver cada pregunta, para no romper el ritmo de la actividad. Si el tiempo de respuesta del Kahoot es excesivamente largo existe el riesgo de la pérdida de atención por parte del alumno y posible alboroto de la dinámica de aula. Otro elemento a tener en cuenta es el nivel de dificultad de las cuestiones planteadas. El hecho de que las cuestiones planteadas sean muy complejas puede desmotivar al alumno y hacer que no se consiga el objetivo, que es el de detectar deficiencias y corregirlas a tiempo, y no para hacerles “caer en la trampa”. Por último, se cree importante destacar que, si el docente no realiza un seguimiento y control muy ordenado del aula durante el Kahoot! planteado, el clima del grupo puede dificultar la obtención de resultados positivos de esta experiencia.

En cuanto a aspectos de utilización de Kahoot revelados en la experiencia docente se destacan dos hechos negativos. Por una parte, la limitación de caracteres en el redactado del enunciado de las preguntas y las respuestas que puede dificultar su planteamiento y por otra parte, el riesgo de salir de la aplicación por parte del alumno al tocar alguna tecla del dispositivo móvil por error. Se recomienda apuntar siempre el Game PIN en la pizarra para que el alumno pueda volver a entrar rápidamente en el juego si ha salido por error.

\section{Conclusiones}

La utilización de los dispositivos móviles en el aula es una práctica cada vez más habitual en la enseñanza superior. En este trabajo se han revisado 30 herramientas de gamificación disponibles en el mercado para ser utilizadas a través de dispositivos móviles y se ha propuesto una clasificación en 6 categorías según sus objetivos educacionales o ámbito de utilidad (Plataformas de gestión del aula, Videojuegos, Unidades de estudio, Compartir presentaciones y contenidos, Edición de vídeos educacionales y Concursos de preguntas y respuestas). En el trabajo también se ha descrito una propuesta metodológica de gamificación a través del uso de una de estas herramientas, Kahoot!.

Los resultados de esta experiencia han mostrado un gran aumento en el seguimiento general de los contenidos del curso que implica una importante mejora en el interés y entusiasmo de los alumnos atribuible a esta experiencia de aprendizaje gamificada. También se ha detectado un mayor grado de compromiso y proactividad para aclarar conceptos y resolver dudas. Esta evolución reafirma el importante cambio logrado en el interés por parte de los alumnos a asistir a clase y es muy alentadora para continuar trabajando en esta línea ya que los alumnos, no solo han asistido más a clase sino que han manifestado una mayor disposición a participar en discusiones y debates de una manera voluntaria y proactiva. El rendimiento académico de los alumnos en base a la nota final que han obtenido de la asignatura se sigue situando dentro de los parámetros habituales de la asignatura y no ha experimentado cambios significativos por el hecho de introducir esta experiencia de gamificación.

En futuros estudios se propone profundizar en la metodología para la evaluación de la propuesta de gamificación planteada en base a la analítica de aprendizaje, learning analytics, para realizar un seguimiento más ajustado de su impacto en el proceso de enseñanza-aprendizaje del estudiante. Así, se piensa que el análisis de la evolución de los resultados obtenidos por los alumnos en los Kahoot!\#1 y Kahoot!\#2 permitiría analizar de una manera más ajustada el grado de adquisición del conocimiento de los alumnos en base a esta propuesta metodológica.

\section{Referencias}

ALBERTA (2012) Bring your own device ISBN 978-14601-0338-8 (Online) Disponible vía: http://education. alberta.ca/admin/technology/research.aspx Consultado: $17 / 01 / 2017$

ALEJANDRE J L (2017) Buenas prácticas en la docencia universitaria con apoyo de TIC. Experiencias en 2016 Prensas de la Universidad de Zaragoza, ISBN 8416933839

AREA M, GONZÁLEZ C, MORA C (2015) Más allá de los libros de texto: Textos digitales educacionales y Gamificación de los materiales de aprendizaje Disponible vía: http://manarea.webs.ull.es/wp-content/ uploads/2014/01/digital-text-area-gonzalez-mora.pdf Consultado: 23/02/2016

ARJONA J, GAMIZ V (2013) Revisión de opciones para el uso de la plataforma Moodle en dispositivos Móviles Revista de Educación a Distancia, XII(37), pp. 1- 15

BARATA G, GAMA S, JORGE J, GONÇALVES D (2013) Engaging Engineering Students with Gamification. Disponible vía: https://www.researchgate.net/ publication/309050128

BENNETT S, MATON K, KERVIN L (2008) The 'digital natives' debate: A critical review of the evidence. British Journal of Educational Technology, 39 (5), pp. 775-786. doi: $10.1111 /$ j.1467-8535.2007.00793.x

CARNEVALE D (2005) "Run a class like a game show: Clickers keep students involved”. Chronicle of Higher Education, 51 (42). 
CORTIZO JC, CARRERO F, MONSALVE B, VELASCO A, DÍAZ L, PÉREZ J (2011) Gamificación y Docencia: Lo que la Universidad tiene que aprender de los Videojuegos, VIII Jornadas Internacionales de Innovación Universitaria. Retos y oportunidades del desarrollo de los nuevos títulos en educación superior. Universidad Europea de Madrid.

DALE E (1969) Audiovisual methods in teaching New York: Dryden Press

DETERDING S, DIXON D, KHALED R, NACKE L (2011) From game design elements to gamefulness: Defining "gamification". Proceedings of the 15th International Academic MindTrek Conference: Envisioning Future Media Environments, pp. 9-15. ISBN: 978-1450308168. doi: $10.1145 / 2181037.2181040$

EDUCACIÓN 3.0 (2017) 15 herramientas para evaluar a los estudiantes Disponible vía: https://www.educaciontrespuntocero.com/recursos/herramientas-evaluar-estudiantes/35095.html Consultado: 12/12/2017

EDUCACIÓN 3.0 (2018) 20 herramientas de gamificación para clase que engancharán a tus alumnos Disponible vía: https://www.educaciontrespuntocero.com/ recursos/herramientas-gamificacioneducacion/33094.html Consultado: 03/03/2018

ESPESO P (2018) Paso a paso: cómo crear un Kahoot! para usar en clase Disponible vía: https://www.educaciontrespuntocero.com/recursos/tutorial-crear-un-kahoot-para-clase/40146.html Consultado: 08/09/2018

FERNÁNDEZ I (2015) Juego serio: gamificación y aprendizaje Disponible via: http://www.centrocp.com/ comunicacion-y-pedagogia-281-282-gamificacion/ Consultado: 23/03/2017

FIES C, MARSHALL J (2006) Classroom response systems: A review of the literature. Journal of Science Education and Technology, 15 (1), pp. 101-109. doi: 10.1007/s10956-006-0360-1

GERSTEIN J (2014) "Moving from Education 1.0 Through Education 2.0 Towards Education 3.0". Experiences in Self-Determined Learning, pp. 83-98.

GONZÁLEZ N, SALCINES I (2015) El Smatphone en los procesos de enseñanza- aprendizaje-evaluación en Educación Superior. Percepciones de docentes y estudiantes. RELIEVE, 21 (2), art. M3. doi: 10.7203/relieve.21.2.7480

GUERRERO C, JAUME A, JUIZ C, LERA I (2016) Use of Mobile Devices in the Classroom to Increase Motivation and Participation of Engineering University Students IEEE Latin America Transactions, 14(1), pp. 411 - 416
GUILLÉN V, ALESON M (2012) Serious games and learning effectiveness: The case of It's a Deal! Computers \& Education, 58(1), 435-448. doi:10.1016/j. compedu.2011.07.015

HAMARI J, KOIVISTO J, SARSA H (2014) Does gamification work? - A literature review of empirical studies on gamification. Proceedings of the Annual Hawaii International Conference on System Sciences, pp. 3025-3034. ISBN: 978-147992504-9 doi: 10.1109/HICSS.2014.377

IGLESIAS A (2016) "Queremos que los Consejos de Dirección aprendan jugando” Disponible vía: http://www. ticbeat.com/entrevistas/johan-brand-Kahoot!-queremos-que-los-consejos-de-direccion-aprendan-jugando/ Consultado: 20/01/2017

KAPP, K M (2012) The gamification of learning and instruction: game-based methods and strategies for training and education. San Francisco: Pfeiffer.

LÓPEZ FA, SILVA MM (2016) Factores que inciden en la aceptación de los dispositivos móviles para el aprendizaje en educación superior. Estudios sobre educación, 30, pp. $175-195$

MANDERNACH BJ, DONNELLI-SALLEE E, DAILEY-HEBERT, A. (2011) Assessing course student engagement. En: MILLER R, AMSEL E, KOWALEWSKI BM, BEINS BB, KEITH KD, PEDEN BF (Eds.), Promoting Student Engagement: Techniques and Opportunities, pp. 277-281, Society for the Teaching of Psychology, Division 2, American Psychological Association.

MARGALEF L (2014) Formative assessment in higher education: Teachers' resistance and paradox Education XXI Open Access 17(2), pp. 35-55

MAYO, M J (2007) Games for science and engineering education. Communications of the ACM, 50(7), pp. 30-35. doi: $10.1145 / 1272516.1272536$

MORRIS C, CHIKWA G (2013) Screencasts: how effective are they and how do students engage with them? Act. Learn. High Educ. 15(1), pp. 25-37

MOYA M, CARRASCO M, JIMÉNEZ M, RAMÓN A, SOLER C, VAELLO M (2016) El aprendizaje basado en juegos: experiencias docentes en la aplicación de la plataforma virtual "Kahoot". En: Actas XIV Jornadas de Redes de Investigación en Docencia Universitaria, Alicante

NAVARIDAS F, SANTIAGO R, TOURÓN J (2013) Valoraciones del profesorado del área de Fresno sobre la influencia de la tecnología móvil en el aprendizaje de sus estudiantes Rev. Electrónica de Investigación y Evaluación Educativa 19(2), pp.1-20 
PAPASTERGIOU, M. (2009) Digital Game-Based Learning in high school Computer Science education: Impact on educational effectiveness and student motivation. Computers and Education, 52 (1), pp. 1-12. doi: 10.1016/j. compedu.2008.06.004

PLUMP C, LAROSA J (2017) Using Kahoot! in the Classroom to create engagement and active learning: GBL Technology Solutions for eLearning Novices. Management Teaching Review doi:10.1177/2379298116689783

POLITES, J (2017) Why the Gamification Market Industry Will Grow 500\% to $\$ 11$ Billion by 2020 Disponible vía: https://blog.atrivity.com/why-gamification-industry-will-grow-to-11-billion-by-2020 Consultado: 02/03/2018

PRENSKY, M. (2001) Digital Games-based Learning. McGraw Hill New York, London.

PRIETO MARTIN A, et al (2014) Experiencias de aplicación de estrategias de gamificación a entornos de aprendizaje universitario. ReVision, 7(2), pp. 76-92

RAFART A, BIKFALVI A, SOLER J, PRADOS F, POCH J (2017) Journal of Technology and Science Education 7(2), pp. 203-220 - Online ISSN: 2013-6374 - Print ISSN: 2014-5349 doi.org/10.3926/jotse.252

RAMIREZ, J.L. (2014) GAMIFICACION: Mecánicas de juegos en tu vida personal y profesional ISBN 10: 8494127268 / ISBN 13: 9788494127267 Editorial: Servicio Comercial del Libro

RAMOS, A. I., HERRERA, J. A., \& RAMÍREZ, M. S. (2010) Desarrollo de habilidades cognitivas con aprendizaje móvil: un estudio de casos. Revista Científica de Educomunicación, XVII(34), pp. 201-209.

ROIG R, MIRA J, CARRERES A, BUADES N, ALFOSEA J, DE CALIDAD V (2016) Investigación e Innovación Educativa en Docencia Universitaria. Retos, Propuestas y Acciones. Alicante: Universidad de Alicante, Instituto de Ciencias de la Educación

ROMERA, C (2018) Gamificación en las aulas. Disponible vía: https://vanadis.es/gamificacion-en-las-aulas/ Consultado el 18/06/2018

ROSALES MM (2014) Proceso evaluativo: evaluación sumativa, evaluación formativa y Assesment: su impacto en la educación actual. Actas del Congreso Iberoamericano de Ciencia, Tecnología, Innovación y Educación ISBN: 978-84-7666-210-6 - Artículo 662
SELLÉS M, SÁNCHEZ S, PÉREZ E (2016) Aplicación de la plataforma KAHOOT en asignaturas de Ingeniería de Fabricación, Congreso In-Red 2016 UPV, 7 y 8 de julio de 2016 doi:10.4995/INRED2016.2016.4400

SHELL J, et al (2013) Catalyzing learner engagement using cutting-edge classroom response systems in higher education. Cutting-edge Technologies in Higher Education, 6, pp.233-261.

TICBEAT (2016) Johan Brand (Kahoot!): "Queremos que los Consejos de Dirección aprendan jugando”. Disponible vía: http://www.ticbeat.com/entrevistas/johan-brand-Kahoot!-queremos-que-los-consejos-de-direccion-aprendan-jugando/ Consultado: 25/09/2018

TOURÓN J, SANTIAGO R, DÍEZ A (2014) The flipped classroom. Como convertir la escuela en un espacio de aprendizaje. [The flipped classroom. How to make the school a learning environment]. Digital-Text, Barcelona

VILLEGAS A (2016) El Móvil en el Aula - Ideas, Ventajas, Retos y Posibilidades Disponible vía: http://www.e-historia.cl/e-historia/movil-aula-ideas-ventajas-retos-posibilidades/ Consultado: 17/01/2017

WANG A, ZHU M, SÆTRE R (2016) The Effect of Digitizing and Gamifying Quizzing in Classrooms. Proceedings of the European Conference on Games-based Learning, January, pp. 729-737.

WERBACH K, HUNTER, D (2012) For the win: How game thinking can revolutionize your business. Pennsylvania: Wharton Digital Press

WILLIS J (2010) Current impact of neuroscience in teaching and learning Mind, Brain, and Education: Neuroscience Implications for the Classroom, Edited by David Sousa, Solution Tree

ZARZYCKA E (2014) Kahoot it or not? Can games be motivating in learning grammar? Teach. Engl. Technol. 16(3), pp. 17-36

ZICHERMANN G, CUNNINGHAM C (2011). Gamification by Design: Implementing Game Mechanics in Web and Mobile Apps. O'Reilly Media, Inc. 